\title{
OPEN Assessing cost-effectiveness of hepatitis $C$ testing pathways in Georgia using the Hep C Testing Calculator
}

\author{
Madeline Adee ${ }^{1,6}$, Yueran Zhuo ${ }^{1,2,3,6}$, Huaiyang Zhong ${ }^{1,2}$, Tiannan Zhan ${ }^{1}$, Rakesh Aggarwal ${ }^{4}$, \\ Sonjelle Shilton ${ }^{5,7}$ \& Jagpreet Chhatwal ${ }^{1,2,7 凶}$
}

The cost of testing can be a substantial contributor to hepatitis C virus (HCV) elimination program costs in many low- and middle-income countries such as Georgia, resulting in the need for innovative and cost-effective strategies for testing. Our objective was to investigate the most cost-effective testing pathways for scaling-up HCV testing in Georgia. We developed a Markov-based model with a lifetime horizon that simulates the natural history of HCV, and the cost of detection and treatment of HCV. We then created an interactive online tool that uses results from the Markov-based model to evaluate the cost-effectiveness of different HCV testing pathways. We compared the current standardof-care $(\mathrm{SoC})$ testing pathway and four innovative testing pathways for Georgia. The SoC testing was cost-saving compared to no testing, but all four new HCV testing pathways further increased QALYs and decreased costs. The pathway with the highest patient follow-up, due to on-site testing, resulted in the highest discounted QALYs (124 QALY more than the SoC) and lowest costs $(\$ 127,052$ less than the SoC) per 10,000 persons screened. The current testing algorithm in Georgia can be replaced with a new pathway that is more effective while being cost-saving.

Chronic hepatitis $\mathrm{C}$ virus (HCV) infection is a global health problem that affects about 71 million people worldwide ${ }^{1}$. Of these, only $19 \%$ knew their infection status in $2017^{1}$. In many countries, HCV-related disease burden and deaths have been steadily increasing, despite recent advances in HCV treatment ${ }^{1}$. The highly effective direct-acting antivirals (DAAs) that became available from 2015 onwards can achieve high rates of sustained virologic response (SVR), a surrogate for cure ${ }^{2}$. However, a huge majority-more than $80 \%$ - of HCV patients remain undiagnosed and therefore are unable to avail the benefits of improved survival and quality of life provided by DAAs ${ }^{1}$.

The World Health Organization (WHO) recently launched a global strategy for elimination of HCV as a public health threat by the year 2030 . This strategy aims to reduce HCV incidence by $80 \%$ and HCV-related mortality by $65 \%^{3}$. To reach this goal, the WHO estimates that by 2030 at least $90 \%$ of people with HCV need to be diagnosed, with a treatment rate of at least $80 \%$ among all treatment-eligible people with $\mathrm{HCV}^{3}$.

However, most countries do not have an HCV elimination strategy. In particular, for low- and middle-income countries (LMIC), which have limited resources but high HCV prevalence rates ${ }^{4}$, it is important to develop a cost-effective HCV elimination strategy. Given that the price of DAAs is low in most LMICs, the cost of testing can be a substantial contributor to the cost of HCV elimination ${ }^{5}$.

Georgia, a LMIC country, has a high HCV disease burden with prevalence of $5.4 \%$ in adults ${ }^{6,7}$, and has launched a national program to eliminate HCV. The Georgian health care system is largely private, but the national HCV elimination program formed a partnership between private and public institutions with a cost sharing model-with treatment provided for free through a donation from Gilead ${ }^{8}$. However, a recent study concluded that to achieve the goal of eliminating HCV as a public health threat in Georgia, innovative, simple, and cost-effective strategies are needed to scale-up HCV testing ${ }^{9,10}$. To help address this issue, the Foundation for Innovative Diagnostics (FIND) has proposed new testing pathways for HCV in Georgia.

\footnotetext{
${ }^{1}$ Massachusetts General Hospital, Boston, MA, USA. ${ }^{2}$ Harvard Medical School, Boston, MA, USA. ${ }^{3}$ College of Business, Mississippi State University, Mississippi State, MS, USA. ${ }^{4}$ Jawaharlal Institute of Postgraduate Medical Education and Research, Puducherry, India. ${ }^{5}$ Foundation for Innovative New Diagnostics, Geneva, Switzerland. ${ }^{6}$ These authors contributed equally: Madeline Adee and Yueran Zhuo. ${ }^{7}$ These authors jointly supervised this work: Sonjelle Shilton and Jagpreet Chhatwal. ${ }^{\circledR}$ email: jagchhatwal@mgh.harvard.edu
} 


\begin{tabular}{|l|l|}
\hline Parameter & Value \\
\hline Age (median), years ${ }^{15}$ & 39 \\
\hline Fibrosis score distribution $^{6}$ & \multicolumn{2}{|l|}{} \\
\hline F0 & $38 \%$ \\
\hline F1 & $32 \%$ \\
\hline F2 & $13 \%$ \\
\hline F3 & $10 \%$ \\
\hline F4 & $7 \%$ \\
\hline Sex distribution & \\
\hline Male & $50 \%$ \\
\hline Female & $50 \%$ \\
\hline Virus genotype distribution & $6,7,16 *$ \\
\hline G1 & \multicolumn{2}{|l}{} \\
\hline G2 & $40 \%$ \\
\hline G3 & $24 \%$ \\
\hline G4 & $34 \%$ \\
\hline
\end{tabular}

Table 1. Baseline population characteristics among HCV-infected persons in Georgia. HCV hepatitis C virus, $F$ METAVIR fibrosis score, $G$ genotype. ${ }^{*} \mathrm{HCV}$ genotypes 5 and 6 were not considered because of their rarity in Georgia. All the distributions in this table, including fibrosis score, sex and genotype were taken as independent of each other and assumed to have no dependencies.

The objective of this study was to evaluate the long-term cost-effectiveness of different HCV testing pathways in Georgia. We also developed an interactive online tool to assess and compare the health-related and economic outcomes of different pathways under different settings of HCV epidemic, patient flow and costs.

\section{Methods}

Overview. We utilized a state-transition model, MATCH (Markov-based Analyses of Treatments for Chronic Hepatitis C), which simulates HCV disease progression. Natural history outcomes from this model have been validated previously ${ }^{1-13}$. We adapted this model to simulate the epidemiology of HCV in Georgia (MATCHGeorgia), and extended the model to evaluate the cost-effectiveness of several innovative HCV testing pathways for Georgia. The model was developed following the principles on economic analyses with respect to viral hepatitis recommended by the $\mathrm{WHO}^{14}$. Using the results from this model, we also developed an interactive online tool, the Hep C Testing Calculator (www.hepccalculator.org), that allows users to compare the cost-effectiveness of different testing pathways for Georgia by entering key model inputs as applicable to the local situation.

Baseline population characteristics. We ran the model for a general population cohort of 10,000 adults in Georgia, with an HCV antibody prevalence of $2 \%$ in the base case ${ }^{6}$, and the percentage of viremic infection among HCV antibody positive people of $75 \%$. The baseline characteristics of HCV patients were determined by the different combinations of sex, HCV genotype, and METAVIR fibrosis stage observed in HCV patients in Georgia (Table 1). All HCV-infected patients were considered treatment-naïve because treatment coverage, until recently, had been very limited in Georgia. We assumed an average baseline age of 45 years. No human subjects were involved in this research.

Testing pathways. We simulated five testing pathways for HCV diagnosis and monitoring. Among these pathways, one represents the standard of care (SoC) in Georgia, whereas the other four represent innovative testing pathways proposed by FIND and initiated under the HEAD-Start Harm Reduction study ${ }^{17}$. Each pathway consists of several sequential testing stages including initial screening, confirmation of presence of HCV RNA, liver staging, and treatment response (Fig. 1). Pathways differ in the testing technologies used (including sensitivity and specificity of each test) and in locations where each test is performed-on-site, specimen collected onsite and then sent to a laboratory, or at another location that the patient must travel to. All pathways use on-site $\mathrm{HCV}$-antibody rapid diagnostic testing for screening. Confirmation is done using either HCV-RNA testing or HCV core-antigen testing. Liver staging consists of two phases of testing using either Fibroscan or APRI/FIB4, and for some pathways phase 2 is completed only for patients with METAVIR fibrosis score of 4 . Treatment is monitored using RNA or biochemical testing, and all pathways use RNA testing for SVR evaluation.

DAA treatment regimens and efficacy. Patients with viremic HCV infection who made it through the second liver-staging test in the pathway (or the first, if only one was done) were eligible to receive DAA-based treatment. The DAA regimens used in the model were determined by individual patient's liver fibrosis stage. Data about the regimens, including their efficacy in different scenarios, were obtained from clinical trials ${ }^{18-20}$ (Supplementary Table S1). 


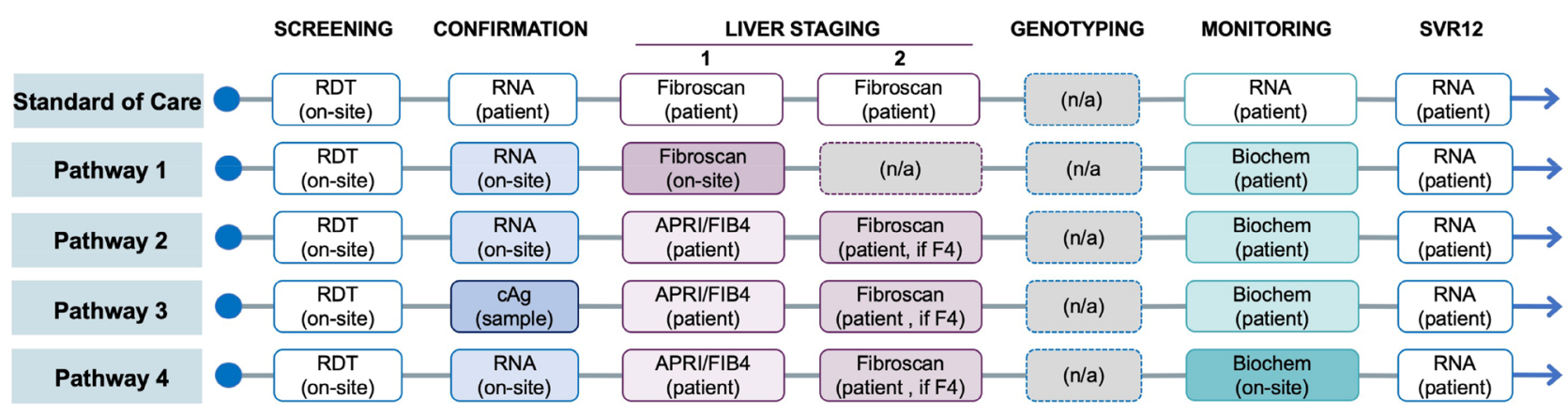

Figure 1. Patient flow under the current standard-of-care testing pathway and innovative hepatitis $\mathrm{C}$ testing pathways in Georgia. Abbreviations: RDT, rapid diagnostic test; RNA, ribonucleic acid test; cAg, core antigen test; APRI/FIB4, aspartate aminotransferase (AST)-to-platelet ratio index (APRI)/fibrosis-4 index; F4, METAVIR fibrosis score of 4.

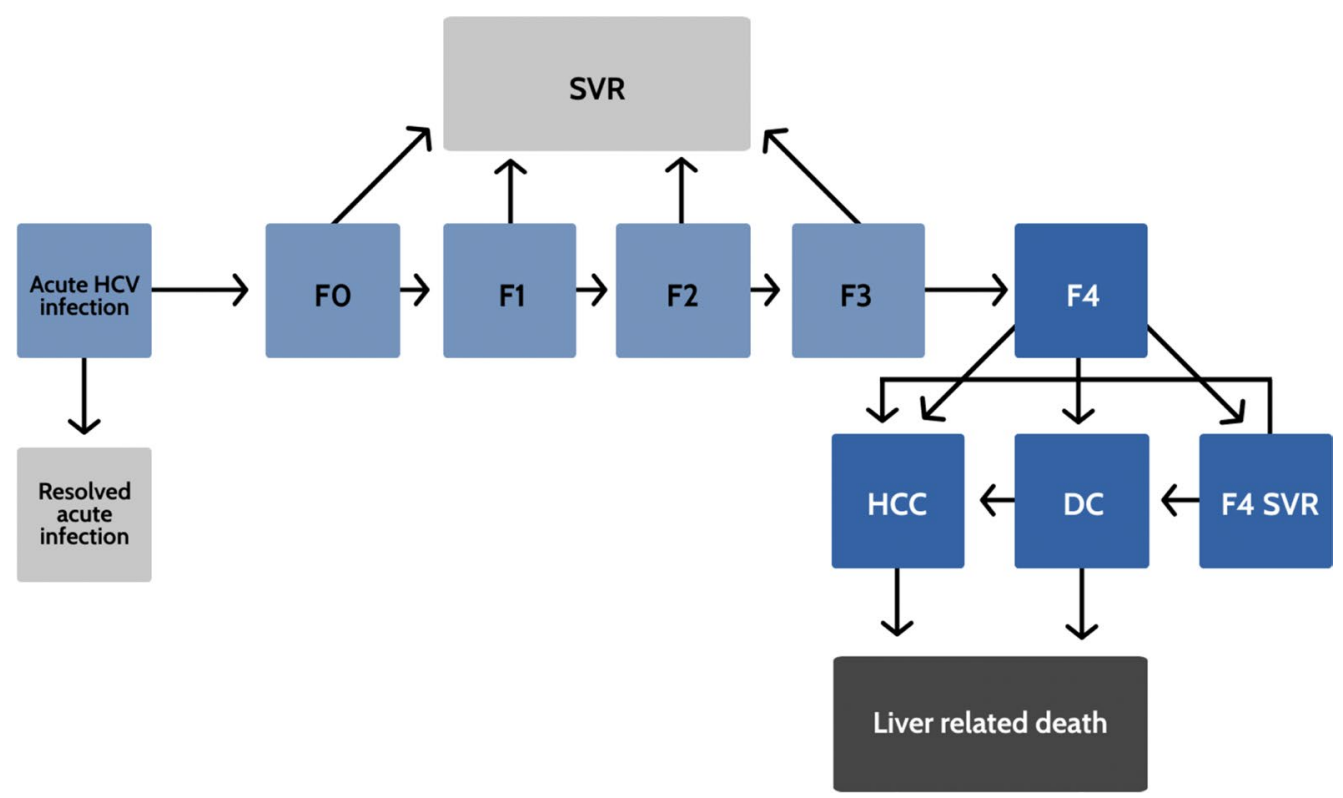

Figure 2. Model schematic of the natural history of hepatitis C virus in MATCH-Georgia model. Abbreviations: SVR, sustained virologic response; F0-F4, METAVIR fibrosis score; DC, decompensated cirrhosis; HCC, hepatocellular carcinoma; F4-SVR, sustained virologic response achieved at fibrosis stage 4.

Disease progression. Patients with HCV followed the natural history of HCV disease progression, defined as Markov health states in the MATCH-Georgia model (Fig. 2). Each patient started in a METAVIR liver fibrosis state of F0-F4. At the end of each simulation cycle (defined as one week), the patient could remain in the same state, progress into a more severe adjacent state of fibrosis, decompensated cirrhosis (DC), hepatocellular carcinoma (HCC), or liver-related death (LRD) or background mortality. Patients in the F0-F3 states who achieved SVR were considered cured and followed background mortality from that point on. However, patients who achieved SVR in the F4 state could still progress to DC, HCC and LRD states, though at a lower rate than F4 patients who had not achieved SVR ${ }^{21}$. Patients who fail treatment were assumed to continue to progress at the original rate. The fibrosis progression rates from F0 to F4 were based on a published meta-regression analysis ${ }^{22}$; progression rates from cirrhosis to DC and HCC are from published observational studies ${ }^{23,24}$. The liver-related mortality rates from DC and HCC were also derived from a published study ${ }^{25}$. The model did not include liver transplantation as a state due to the rarity of this procedure in Georgia.

Quality of life weights. The model assigns quality-of-life (QoL) weights to each health state. All HCVrelated QoL weights were derived from published studies ${ }^{26-28}$. People without HCV were assigned QoL weights according to their sex and age, and for patients who achieved SVR, the QoL weights of the health states were assumed to be equivalent to that of the non-HCV-infected general population ${ }^{26}$. However, if patients who achieved SVR progressed to DC or HCC, then the QoL weights of the corresponding state was applied. The adverse effect of anemia on quality of life during the treatment period was also considered, by applying an 
anemia multiplier. All HCV-related and normal QoL weight values are summarized in Table 2. The use of QoL weights allows us to present health-related outcomes as quality-adjusted life years (QALYs).

Costs. The MATCH-Georgia model considered HCV diagnosis costs, DAA-based treatment costs and HCV disease management costs. All costs were considered from a healthcare payer's perspective.

The HCV testing costs consisted of the cost incurred at each step of the testing pathway, which included not only the costs for conducting laboratory tests, but also those for specimen shipment or of patient travel, as required, as these are integral parts of each testing pathway (Table 2). These costs were estimated from FIND HEAD-Start Georgia study ${ }^{29}$. Patients who failed to follow up in the next testing stage no longer incurred any further testing costs and did not receive DAA treatment. Free DAA medicines are now available for Georgia HCV patients through contracts with the pharmaceutical companies ${ }^{30}$, however we added $\$ 100$ per person treated as an operational expense.

We estimated annual healthcare costs associated with HCV disease management using the World Health Organization's CHOosing Interventions that are Cost Effective (WHO-CHOICE) tool ${ }^{34}$ (Table 2). For that, we first extracted inpatient and outpatient primary costs from WHO-CHOICE and took the weighted average of cost per inpatient visit and cost per outpatient visit for each $\mathrm{HCV}$-associated states in the United States; inpatient visits accounted for $38 \%$ of healthcare encounters for F0-F4 patients, $43 \%$ for compensated cirrhosis patients, $66 \%$ for DC patients, and 55\% for HCC patients ${ }^{35}$. We then estimated the ratio of the above costs in Georgia to United States and, finally, estimated Georgia-specific costs by multiplying this ratio with costs in the United States $^{35}$. To account for differences in medical practices between Georgia and the United States, we considered a wide range in costs in the sensitivity analysis.

Model outcomes. For each pathway, we projected average QALYs, total cost, and cumulative incidence of DC, HCC, and HCV-related deaths. We also estimated the testing costs per case treated and incremental costeffectiveness ratio (ICER) of each pathway. A lifetime horizon was used, and all future costs and QALYs were discounted at $3 \%$ per year.

Interactive tool. We also developed an interactive online tool using R Shiny that allows users to change certain inputs and evaluated the comparative effectiveness and cost-effectiveness of different diagnostic testing pathways. In this tool, users can change the population cohort size, screening rate, prevalence rates (of anti-HCV antibody in the population and of viremia among HCV-seropositive persons), and patient/client follow up rate for each step in a testing pathway-with the ability to add custom testing pathways. The users can also change costs for DAAs, each diagnostic test, patient/client travel, and specimen shipment.

Once parameters are changed, the tool shows updated results for the total expected QALYs, costs, and disease burden for each of the testing pathways. It also calculates the ICERs of the testing pathways by comparing their QALYs and costs, to assist users in identifying the most cost-effective testing pathways. A screenshot of the interactive tool is provided in Supplemental Figure S1. The tool is still being expanded and can be accessed at hepccalculator.org.

Sensitivity analysis. We performed both deterministic and probabilistic sensitivity analyses to evaluate the effect of variations in model inputs on the cost-effectiveness of the testing pathways. These inputs included state transition probabilities, QoL weights, medical and disease management costs, diagnostic test costs, patient travel/sample shipping costs, and patient follow-up rates. Both the one-way and probabilistic sensitivity analyses also included HCV demographic parameters such as HCV prevalence and viremic rate in HCV antibody positive people. The ranges of all model inputs used for sensitivity analyses, and distribution used for the probabilistic sensitivity analysis, are defined in Table 2 .

\section{Results}

Cost-effectiveness of HCV testing pathways. Compared with no screening, HCV screening under the SoC increased discounted QALYs by 332 per 10,000 people screened and decreased costs by US $\$ 290,942$ (Table 3). All the four new HCV testing pathways (Pathways 1-4; Fig. 1) further increased QALYs and decreased costs. Pathway 1-on-site rapid diagnostic test for HCV antibody followed by on-site HCV-RNA confirmatory test, on-site Fibroscan for liver disease staging of chronic HCV patients, sample transportation for genotype testing, and on-site HCV-RNA test for assessment of treatment response-resulted in the highest discounted QALYs of 205,702 (124 QALY more than that under the SoC) and lowest costs of \$142,939 (\$127,052 less than that under SoC) per 10,000 persons screened. Compared with other pathways, Pathway 1 was cost-saving. The testing-related cost per HCV case treated for the SoC was $\$ 289$ and for Pathway 1 was $\$ 139$. Pathways 2, 3, and 4 all had higher total costs as well as higher testing costs per patient treated.

Clinical efficacy of testing pathways. The diagnosis rate-defined as the percentage of people with viremic $\mathrm{HCV}$ who were eventually diagnosed-of the SoC was $79.2 \%$; by contrast, the diagnosis rate of Pathway 1 was $88 \%$. Patients lost before initiating treatment accounted for a bigger difference between the percent of viremic patients treated, with only $64.2 \%$ of viremic patients treated in the SoC scenario but $88 \%$ of viremic patients treated in Pathway 1. Under SoC, 84 people needed to get antibody screening on average to diagnose one additional HCV-viremic case, while under Pathway 1 this number was 76. All new HCV testing pathways improved the HCV diagnosis rate. 


\begin{tabular}{|c|c|c|c|c|}
\hline Parameter Name & Base Case & Low & High & Distribution \\
\hline \multicolumn{5}{|l|}{ Testing pathway parameters (costs in USD) } \\
\hline Antibody RDT test unit $\operatorname{cost}^{29}$ & 1.00 & 0.50 & 1.50 & Gamma $(18,0.0556)$ \\
\hline HCV-RNA on-site test unit cost (Genexpert) ${ }^{29}$ & 15.00 & 8.00 & 23.00 & Gamma $(17,0.8824)$ \\
\hline APRI test unit $\operatorname{cost}^{29}$ & 5.00 & 3.00 & 8.00 & Gamma $(14,0.3571)$ \\
\hline Fibroscan test unit $\operatorname{cost}^{29}$ & 33.00 & 17.00 & 50.00 & Gamma $(17,1.9412)$ \\
\hline Biochemical test unit cost ${ }^{29}$ & 10.00 & 5.00 & 15.00 & Gamma $(19,0.5263)$ \\
\hline Genotyping test unit $\operatorname{cost}^{29}$ & 51.00 & 26.00 & 77.00 & Gamma $(18,2.8333)$ \\
\hline HCV-RNA test by referral unit $\cos ^{29}$ & 40.00 & 20.00 & 60.00 & Gamma $(18,2.22222)$ \\
\hline Core antigen $(\mathrm{cAg})$ test unit $\operatorname{cost}^{29}$ & 12.00 & 6.00 & 18.00 & Gamma $(19,0.6316)$ \\
\hline Sample transportation $\operatorname{cost}^{29}$ & 0.37 & 0.19 & 0.56 & Gamma $(18,0.0206)$ \\
\hline Cost of treatment ${ }^{30}$ & 100.00 & 50.00 & 150.00 & Gamma $(19,5.2632)$ \\
\hline HCV antibody prevalence ${ }^{6}$ & $2 \%$ & $1 \%$ & $3 \%$ & Beta $(32,1568)$ \\
\hline Viremic rate in antibody-positive people & $75 \%$ & $50 \%$ & $100 \%$ & Beta $(19,6.3333)$ \\
\hline Target screening rate (assumption) & $90 \%$ & $75 \%$ & $100 \%$ & Beta $(38,4.22222)$ \\
\hline Confirmation test follow-up rate (Expert opinion ${ }^{29}$ ) & $90 \%$ & $75 \%$ & $100 \%$ & Beta $(38,4.22222)$ \\
\hline Liver staging-1 test follow-up rate (Expert opinion ${ }^{29}$ ) & $90 \%$ & $75 \%$ & $100 \%$ & Beta $(38,4.22222)$ \\
\hline Liver staging-2 test follow-up rate (Expert opinion ${ }^{29}$ ) & $90 \%$ & $75 \%$ & $100 \%$ & Beta $(38,4.22222)$ \\
\hline Monitoring test follow up-rate (Expert opinion $\left.{ }^{29}\right)$ & $90 \%$ & $75 \%$ & $100 \%$ & Beta $(38,4.22222)$ \\
\hline SVR12 RNA test follow-up rate (Expert opinion ${ }^{29}$ ) & $90 \%$ & $75 \%$ & $100 \%$ & Beta $(38,4.22222)$ \\
\hline \multicolumn{5}{|l|}{ Transition probabilities (annual) } \\
\hline $\mathrm{F} 0$ to $\mathrm{F}^{22}$ & 0.117 & 0.104 & 0.130 & Beta $(285.98,2158.26)$ \\
\hline $\mathrm{F} 1$ to $\mathrm{F} 2^{22}$ & 0.085 & 0.075 & 0.096 & Beta $(239.77,2581)$ \\
\hline $\mathrm{F} 2$ to $\mathrm{F} 3^{22}$ & 0.120 & 0.109 & 0.133 & Beta $(351.88,2580.45)$ \\
\hline $\mathrm{F} 3$ to $\mathrm{F} 4^{22}$ & 0.116 & 0.104 & 0.129 & Beta $(304.4,2319.73)$ \\
\hline $\mathrm{F} 4$ to $\mathrm{DC}^{23}$ & 0.039 & 0.010 & 0.079 & Beta $(4.87,120.08)$ \\
\hline $\mathrm{F} 4$ to $\mathrm{HCC}^{23}$ & 0.014 & 0.010 & 0.079 & Beta $(0.64,44.75)$ \\
\hline Post F4-SVR to DC ${ }^{21}$ & 0.008 & 0.002 & 0.036 & Beta $(0.87,107.97)$ \\
\hline Post F4-SVR to HCC ${ }^{21}$ & 0.005 & 0.002 & 0.013 & Beta $(3.28,653.57)$ \\
\hline DC to $\mathrm{HCC}^{24}$ & 0.068 & 0.030 & 0.083 & Beta $(24.48,335.51)$ \\
\hline DC (year 1) to death from liver disease ${ }^{24}$ & 0.182 & 0.065 & 0.190 & Beta $(27.56,123.89)$ \\
\hline DC $(1+$ years $)$ to death from liver disease $e^{24}$ & 0.112 & 0.065 & 0.190 & Beta $(11.29,89.55)$ \\
\hline HCC to liver-related death ${ }^{23}$ & 0.427 & 0.330 & 0.860 & Beta $(5.52,7.41)$ \\
\hline \multicolumn{5}{|l|}{ Health state costs (annual in USD)* } \\
\hline F0-F2 & 62 & 31 & 123 & Gamma $(6,10.3333)$ \\
\hline F3 & 126 & 63 & 253 & Gamma $(5,25.2)$ \\
\hline Compensated cirrhosis & 144 & 72 & 289 & Gamma $(6,21)$ \\
\hline Decompensated cirrhosis & 1496 & 748 & 2993 & Gamma $(17,88)$ \\
\hline Hepatocellular cancer & 2625 & 1413 & 5652 & Gamma $(17.17,154.4118)$ \\
\hline F4 post-SVR & 72 & 36 & 144 & Gamma $(5,14.4)$ \\
\hline \multicolumn{5}{|l|}{ Health state quality-of-life weights } \\
\hline Anemia multiplier $^{31}$ & 0.83 & 0.75 & 0.97 & Beta $(80,16.3855)$ \\
\hline F0-F3 ${ }^{26}$ & 0.93 & 0.84 & 1.00 & Beta $(40,3.0108)$ \\
\hline Compensated cirrhosis (F4) ${ }^{26}$ & 0.90 & 0.81 & 0.99 & Beta $(50,5.5556)$ \\
\hline $\mathrm{DC}^{26}$ & 0.80 & 0.57 & 0.99 & Beta $(12,3)$ \\
\hline $\mathrm{HCC}^{26}$ & 0.79 & 0.54 & 0.99 & Beta $(10,2.6582)$ \\
\hline Post-SVR ${ }^{* * *}$ & 1 & 0.92 & 1 & Beta $(3833.92,3.84)$ \\
\hline \multicolumn{5}{|l|}{ Test sensitivity and specificity } \\
\hline Antibody RDT sensitivity ${ }^{32}$ & $98.0 \%$ & $98.0 \%$ & $100.0 \%$ & Uniform $(0.98,1)$ \\
\hline Antibody RDT specificity ${ }^{32}$ & $100.0 \%$ & $100 \%$ & $100.0 \%$ & Uniform $(1,1)$ \\
\hline HCV-RNA (lab) test sensitivity & $99.8 \%$ & $99.6 \%$ & $100.0 \%$ & Uniform $(0.996,1)$ \\
\hline HCV-RNA (lab) test specificity & $99.7 \%$ & $99.4 \%$ & $100.0 \%$ & Uniform $(0.994,1)$ \\
\hline cAg (lab) test sensitivity ${ }^{33}$ & $93.4 \%$ & $90.10 \%$ & $96.40 \%$ & Beta $(150,10.5996)$ \\
\hline cAg (lab) test specificity ${ }^{33}$ & $98.8 \%$ & $97.40 \%$ & $99.50 \%$ & Beta $(150,1.8219)$ \\
\hline \multicolumn{5}{|l|}{ Sex and age-based normal health utility values ${ }^{26}$} \\
\hline Female, age $<29$ & 0.913 & - & - & - \\
\hline Female, age $30-39$ & 0.893 & - & - & - \\
\hline Continued & & & & \\
\hline
\end{tabular}




\begin{tabular}{|c|c|c|c|c|}
\hline Parameter Name & Base Case & Low & High & Distribution \\
\hline Female, age $40-49$ & 0.863 & - & - & - \\
\hline Female, age $50-59$ & 0.837 & - & - & - \\
\hline Female, age 60-69 & 0.811 & - & - & - \\
\hline Female, age $70-75$ & 0.711 & - & - & - \\
\hline Male, age $<29$ & 0.928 & - & - & - \\
\hline Male, age 30-39 & 0.918 & - & - & - \\
\hline Male, age $40-49$ & 0.887 & - & - & - \\
\hline Male, age $50-59$ & 0.861 & - & - & - \\
\hline Male, age 60-69 & 0.840 & - & - & - \\
\hline Male, age $70-75$ & 0.802 & - & - & - \\
\hline
\end{tabular}

Table 2. Model parameters used in the MATCH-Georgia model. RDT rapid diagnostic tests, $R N A$ ribonucleic acid confirmation test, $A P R I$ aspartate aminotransferase to platelet ratio test, FIB4 fibrosis- 4 test, $c A g$ core antigen test, $S V R$ sustained virologic response, FO-F4 METAVIR fibrosis score, DC decompensated cirrhosis, HCC hepatocellular carcinoma, F4-SVR sustained virologic response achieved at fibrosis stage 4 . ${ }^{*}$ We estimated annual healthcare costs associated with HCV disease management using the World Health Organization's CHOosing Interventions that are Cost Effective (WHO-CHOICE) tool. ${ }^{\star \star}$ For patients experienced anemia during treatment, quality of life was multiplied by this factor. ${ }^{* * *}$ For patients who achieved SVR, the QoL weights of the health states are assumed to be equivalent to that of the non-HCV-infected general population ${ }^{26}$. For patients who achieve SVR at state F4 but further progressed to DC and HCC, their QoL weights were adjusted to those of DC and HCC,respectively.

\begin{tabular}{|l|l|l|l|l|l|l|}
\hline & No screening & Standard of care & Pathway 1 & Pathway 2 & Pathway 3 & Pathway 4 \\
\hline Total cost & $\$ 560,933$ & $\$ 269,991$ & $\$ 142,939$ & $\$ 225,122$ & $\$ 251,769$ & $\$ 225,389$ \\
\hline Disease management & $\$ 560,933$ & $\$ 233,067$ & $\$ 111,080$ & $\$ 196,638$ & $\$ 220,262$ & $\$ 196,638$ \\
\hline Testing & - & $\$ 27,053$ & $\$ 18,315$ & $\$ 17,516$ & $\$ 21,250$ & $\$ 17,783$ \\
\hline Treatment & - & $\$ 9,871$ & $\$ 13,544$ & $\$ 10,968$ & $\$ 10,257$ & $\$ 10,968$ \\
\hline QALYs (total cohort) & 205,246 & 205,578 & 205,702 & 205,615 & 205,591 & 205,615 \\
\hline \% viremic diagnosed & $0.0 \%$ & $79.2 \%$ & $88.0 \%$ & $88.0 \%$ & $82.4 \%$ & $88.0 \%$ \\
\hline \% viremic treated & $0.0 \%$ & $64.2 \%$ & $88.0 \%$ & $71.3 \%$ & $66.7 \%$ & $71.3 \%$ \\
\hline Testing cost per treated pt & - & $\$ 281$ & $\$ 139$ & $\$ 164$ & $\$ 213$ & $\$ 166$ \\
\hline $\begin{array}{l}\text { No. needed to screen to diagnose one HCV } \\
\text { case }\end{array}$ & & 84 & 76 & 76 & 81 & 76 \\
\hline No. needed to screen to prevent one LRD & & 556 & 333 & 456 & 526 \\
\hline Disease Burden & & & & 456 \\
\hline Decompensated cirrhosis & 48 & 20 & 9 & 17 & 19 \\
\hline Hepatocellular carcinoma & 30 & 13 & 6 & 11 & 12 \\
\hline Liver-related deaths (LRD) & 41 & 23 & 11 & 17 \\
\hline
\end{tabular}

Table 3. Comparison of health-related outcomes and economic outcomes of the five screening pathways vs. no screening per 10,000 persons screened. DC decompensated cirrhosis, HCC hepatocellular carcinoma, $L R D$ HCV-caused liver related death. ${ }^{\star}$ The cost for no screening represents the cost of management of $\mathrm{HCV}$ sequelae.

The new pathways also improved clinical outcomes. Compared with the SoC, screening 10,000 people under Pathway 1 would reduce the number of DC cases by 11, HCC by 7, and liver-related deaths by 12 in the lifetime horizon. The number of people needed to be screened (for antibody) to avoid one liver-related death for the SoC was 556, for Pathway 1 was 333, for Pathway 2 and Pathway 4 was 456, and for Pathway 3 was 526.

Sensitivity analyses. Pathway 1 remained cost-saving irrespective of the changes in model parameters. Figure 3 shows the 20 parameters that the model is most sensitive to, including QoL after achieving SVR, QoL of patients in F1-F4 states, probability of disease progression from F4 to DC, and costs of managing DC and HCC. One-way sensitivity analysis results for all parameters are shown in Supplement Table S2. Parameters related to the testing pathways, such as costs of different tests or of patient travel or sample shipping and patient/client follow-up rates had less marked influence on the cost-effectiveness of the testing pathways. For the probabilistic sensitivity analysis, Pathway 1 is the preferred cost-saving option in all scenarios, which is illustrated by the costeffectiveness acceptability curve (Fig. 4). 


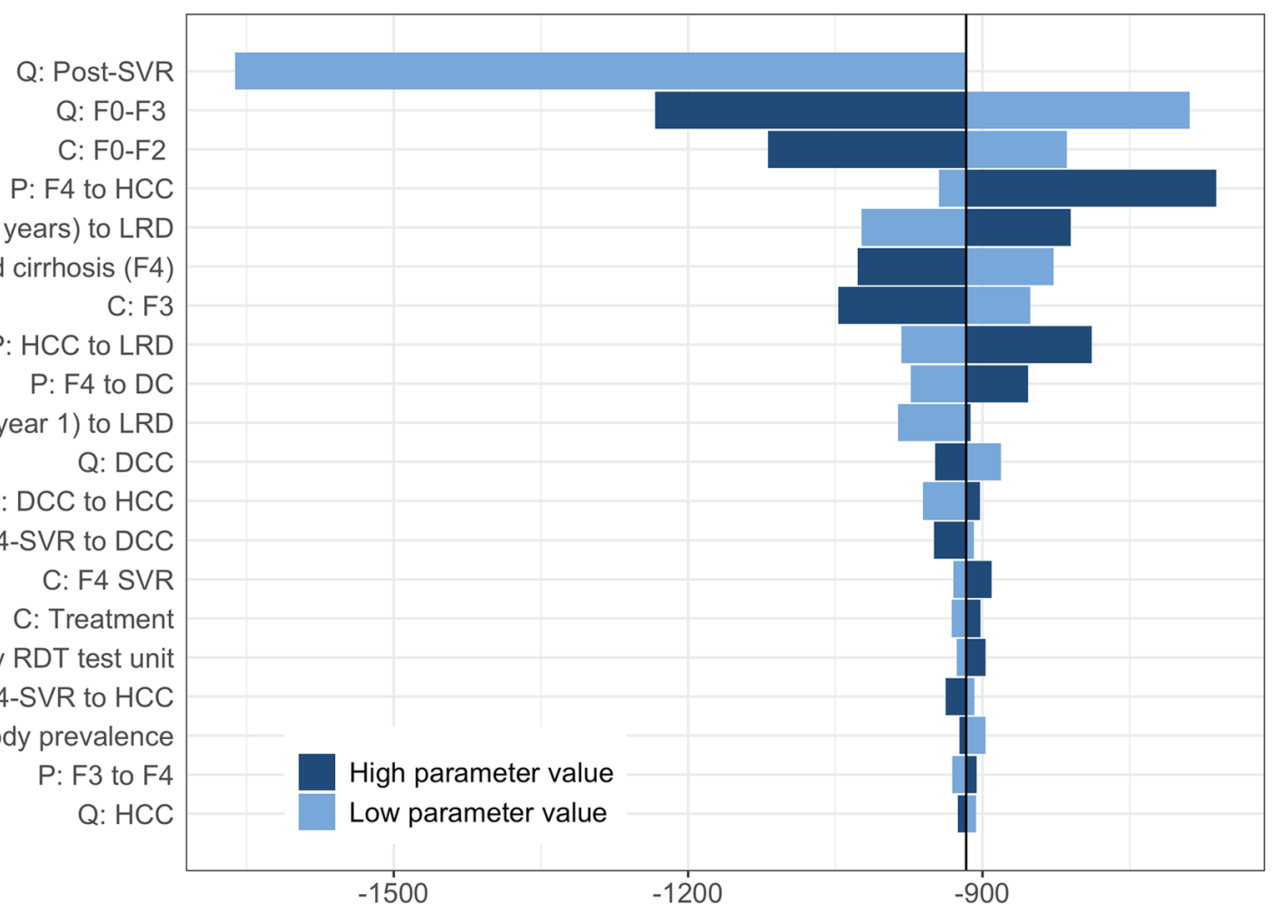

Incremental Cost-Effectiveness Ratio (ICER)

Figure 3. Tornado diagram for one-way sensitivity analysis of incremental cost-effectiveness ratio of Pathway 1 versus no screening strategy. Horizontal bars show the variation in incremental cost-effectiveness ratio (ICER; in USD/QALY) with variation in the value of the parameter. In the parameter names, the prefix ' $\mathrm{C}$ ' represents cost of a health-state, ' $Q$ ' the quality-of-life weight and 'P' the transition probability from one state to the other. Values of ICER below 0 indicate that the treatment is cost-saving. Abbreviations: SVR, sustained virologic response; F0-F4, METAVIR fibrosis score; DC, decompensated cirrhosis; HCC, hepatocellular carcinoma; F4-SVR, LRD, liver related death.

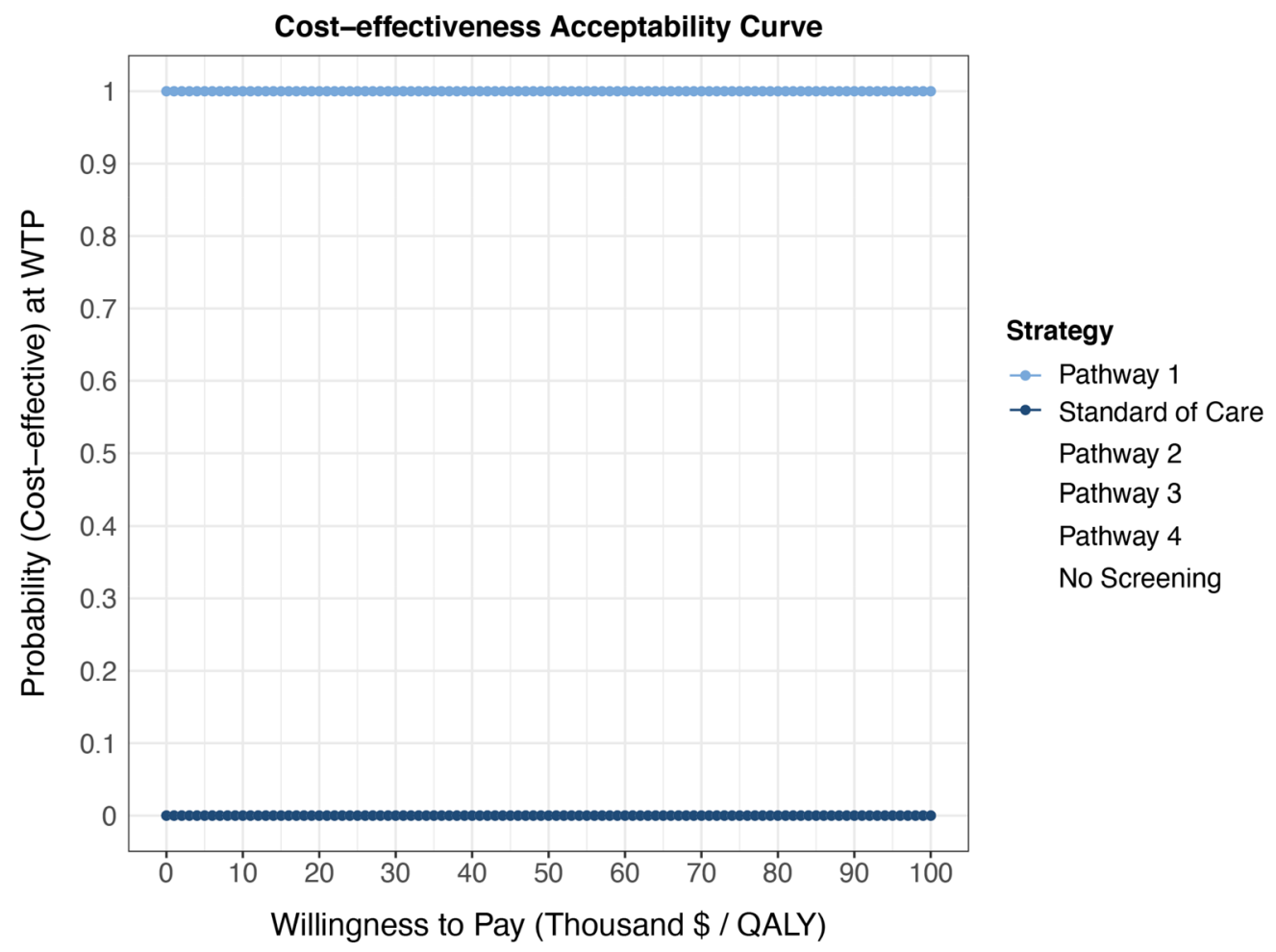

Figure 4. Cost-effectiveness Acceptability Curve of all pathways and no screening strategy. 


\section{Discussion}

The availability of highly effective yet low-priced HCV treatment in LMIC offers an unprecedented opportunity to eliminate HCV as a public health threat. However, the majority of HCV patients remain undiagnosed and hence are not in a position to avail the benefits of new treatments. In this study, we evaluated the cost-effectiveness of five different testing pathways to diagnose and monitor HCV during treatment in Georgia. We found that the pathway using on-site HCV-antibody rapid diagnostic test and HCV-RNA testing, followed by on-site Fibroscan was cost-saving - this pathway would save US $\$ 127,052$ per 10,000 individuals tested (compared with the current standard), while increasing rates of diagnosis and linkage to successful treatment. This pathway would cost $\$ 139$ per HCV case treated and could diagnose $88 \%$ of the viremic cases if scaled-up at the population level.

As pointed out by a recent study, substantial scaling-up of HCV testing and treatment are needed to eliminate $\mathrm{HCV}$ in Georgia ${ }^{9,10}$. However, that study did not evaluate what testing strategies would be cost-effective in Georgia. Therefore, our study fills an important evidence gap. We found that the preferred cost-effective strategies may depend on locally-determined factors, such as the HCV disease epidemiology, costs of different testing methodologies, patient follow-up rates following each visit or procedure, and the on-site availability of diagnostics such a Fibroscan and genotyping, leading to need for patient or specimen transport. We therefore also developed an interactive online-based tool that allows users to change several parameters in the model and identify the cost-effective testing pathway for their localized settings.

As the cost of DAAs has fallen below $\$ 100$ per treatment in Georgia (and other LMICs), the diagnostic cost per HCV case constitutes a substantial portion of HCV care expenses. The cost of diagnosing one HCV case exceeds the cost of HCV treatment in Georgia, which could also be true for many LMICs where low-cost DAAs are available. All countries must domestically finance for these HCV testing and treatment efforts, as there is no global funding mechanism for HCV elimination. This contrasts with HIV, TB, and malaria, for which the Global Fund provides substantial budget annually ${ }^{36}$. Hence, it is very important for LMICs to identify HCV testing pathways that are cost-effective or cost-saving.

Interactive models such as this diagnosis pathway tool and our previously-developed Hep C Treatment Calculator $^{13}$ are important tools to help aid countries, in particular LMICs, in understanding how to best use their existing domestic resources. Since the epidemiology of HCV varies geographically, having a tool that can be fed with location-specific epidemiology and cost inputs could provide countries with the context-specific costeffectiveness estimates needed for their decision making. Our interactive tool takes this one step further. Even within a country, delivery of HCV services to different population groups may require different modes of service provision $^{37}$. This tool can aid in tailoring testing pathway approaches that programs may seek to implement to reach various groups, such as PWID, MSM, age cohorts, regional groups, and others, with differing HCV prevalence and viremia rates, and cost of delivery of each test in specific settings. Hence, our tool could help the public health community to identify and implement the most effective and cost-effective strategy in different settings.

Lastly, it is important to note that the lost-to-follow-up rate remains an important consideration for countrylevel decision makers and program managers. Our analysis shows that the lost-to-follow-up rate has a limited impact on the incremental cost-effectiveness ratio when comparing several testing pathways-however this could be because the pathways have similar set-ups in terms of follow-up rate. An increase in lost-to-follow-up rates will have a similar negative impact on all pathways simultaneously. However, our analysis does not diminish the importance of the lost-to-follow-up rate in HCV testing practice, but rather shows that this issue needs addressing irrespective of the testing pathway chosen.

Our study has some limitations. First, our analysis did not account for continued HCV transmission. Therefore, the benefits of HCV testing, which serves to guide treatment and cure leading to reduced risk of transmission, could be even higher and the optimal pathway could result in even higher cost-savings. Second, since Georgia-specific QoL weights are not available, we used QoL weights from other countries. Our analysis also does not account for different QoL or mortality for specific populations within Georgia, such as people who inject drugs. However, sensitivity analysis suggests that the results remain robust to a wide range of input parameters and that QoL estimates did not change the conclusion of the study. Third, we note that the cost of DAAs in Georgia is negligible due to the contract made with major pharmaceutical companies, a situation that does not apply to most other countries.

In conclusion, our study identified a novel testing pathway to diagnose HCV and monitor its treatment in Georgia with greater effectiveness and found that such a testing pathway would result in cost-savings over the SoC pathway. Our online interactive tool can provide optimal HCV testing pathway under different settings of HCV epidemiology, costs of different tests, patient follow-up rates, and the on-site availability of diagnostics.

Received: 13 November 2020; Accepted: 6 October 2021

Published online: 01 November 2021

\section{References}

1. World Health Organization. Global Hepatitis Report (2017).

2. Falade-Nwulia, O. et al. Oral direct-acting agent therapy for hepatitis C virus infection: A systematic review. Ann. Intern. Med. 166(9), 637-648 (2017).

3. World Health Organization. Combating hepatitis B and C to reach elimination by 2030 (2016).

4. Blach, S. et al. Global prevalence and genotype distribution of hepatitis C virus infection in 2015: A modelling study. Lancet Gastroenterol. Hepatol. 2(3), 161-176 (2017).

5. Chhatwal, J. et al. Assessment of the feasibility and cost of hepatitis C elimination in Pakistan. JAMA Netw. Open 2(5), e193613 (2019).

6. Blach, S. et al. et al. (eds) Polaris Observatory-Global Prevalence of Hepatitis C. Hepatology (Wiley, 2016). 
7. Hagan, L. M. et al. Hepatitis C prevalence and risk factors in Georgia, 2015: Setting a baseline for elimination. BMC Public Health 19(3), 480 (2019).

8. Gvinjilia, L. et al. National progress toward hepatitis C elimination-Georgia, 2015-2016. MMWR 65(41), 1132-1135 (2016).

9. Walker, J. G. et al. Interim effect evaluation of the hepatitis C elimination programme in Georgia: A modelling study. Lancet Glob. Health. 8, e244-e253 (2019).

10. Hutin, Y., Luhmann, N. \& Easterbrook, P. Evaluating the impact of Georgia's hepatitis C elimination plan: Lessons learned for the global initiative. Lancet Glob. Health. 8(2), e163-e164 (2020).

11. Chhatwal, J. et al. Cost-effectiveness of boceprevir in patients previously treated for chronic hepatitis C genotype 1 Infection in the United States. Value Health 16(6), 973-986 (2013).

12. Chhatwal, J., Kanwal, F., Roberts, M. S. \& Dunn, M. A. Cost-effectiveness and budget impact of hepatitis $C$ virus treatment with sofosbuvir and ledipasvir in the United States. Ann. Intern. Med. 162(6), 397-406 (2015).

13. Chhatwal, J. et al. Hep C calculator: An online tool for cost-effectiveness analysis of DAAs. Lancet Gastroenterol. Hepatol. 3(12), 819 (2018).

14. World Health Organization-Viral Hepatitis Strategic Information and Modelling Reference Group. Meeting report | 14-16 June 2016 | WHO headquarters, Geneva, Switzerland (2016).

15. The World Factbook. Country comparisons-median age. https://www.cia.gov/the-world-factbook/field/median-age/countrycomparison2021.

16. Sharvadze, L., Nelson, K. E., Imnadze, P., Karchava, M. \& Tsertsvadze, T. Prevalence of HCV and genotypes distribution in general population of Georgia. Georgian Med. News. 165, 71-77 (2008).

17. U.S. National Library of Medicine: ClinicalTrials.gov. Evaluation of HCV viremia testing approaches among PWID in Georgia (HEAD-Start): NCT03594838 (2020).

18. Afdhal, N. et al. Ledipasvir and sofosbuvir for untreated HCV genotype 1 infection. N. Engl. J. Med. 370(20), 1889-1898 (2014).

19. Nelson, D. R. et al. All-oral 12-week treatment with daclatasvir plus sofosbuvir in patients with hepatitis C virus genotype 3 infection: ALLY-3 phase III study. Hepatology 61(4), 1127-1135 (2015).

20. Kohli, A. et al. Ledipasvir and sofosbuvir for hepatitis $C$ genotype 4: A proof-of-concept, single-centre, open-label phase 2 a cohort study. Lancet Infect. Dis. 15(9), 1049-1054 (2015).

21. Cardoso, A. C. et al. Impact of peginterferon and ribavirin therapy on hepatocellular carcinoma: Incidence and survival in hepatitis C patients with advanced fibrosis. J. Hepatol. 52(5), 652-657 (2010).

22. Thein, H., Yi, Q., Dore, G. \& Krahn, M. Estimation of stage specific fibrosis progression rates in chronic hepatitis $\mathrm{C}$ virus infection: A meta analysis and meta regression. Hepatology 48(2), 418-431 (2008).

23. Fattovich, G. et al. Morbidity and mortality in compensated cirrhosis type C: A retrospective follow-up study of 384 patients. Gastroenterology 112(2), 463-472 (1997).

24. Planas, R. et al. Natural history of decompensated hepatitis C virus-related cirrhosis. A study of 200 patients. J. Hepatol. 40(5), $823-830$ (2004).

25. Wolfe, R., Roys, E. \& Merion, R. Trends in organ donation and transplantation in the United States, 1999-2008. Am. J. Transplant. 10(4p2), 961-72 (2010).

26. Chong, C. A. K. Y. et al. Health-state utilities and quality of life in hepatitis C patients. Am. J. Gastroenterol. 98(3), 630-638 (2003).

27. Hanmer, J., Lawrence, W. F., Anderson, J. P., Kaplan, R. M. \& Fryback, D. G. Report of nationally representative values for the noninstitutionalized US adult population for 7 health-related quality-of-life scores. Med. Decis. Mak. 26(4), 391-400 (2006).

28. Siebert, U. et al. Cost effectiveness of peginterferon-2b plus ribavirin versus interferon-2b plus ribavirin for initial treatment of chronic hepatitis C. Gut 52(3), 425 (2003).

29. Feasibility, acceptability, effectiveness and cost-analysis of models of HCV viremia testing for confirmation and cure among people who inject drugs (PWID): the HEAD-start trial in Georgia. Identifier: NCT03594838. Retrieved from: https://clinicaltrials.gov/ ct2/show/study/NCT03594838 (2019).

30. Reuters. Gilead uses Georgia as free-drug test bed for hepatitis C elimination. 2015. Available from: https://www.reuters.com/artic le/us-health-hepatitis-gilead-georgia/gilead-uses-georgia-as-free-drug-testbed-for-hepatitis-c-elimination-idUSKBN0ND1XU20 150422 (last accessed 31 January 2020) (2015).

31. Wilson, J. et al. A systematic review and economic evaluation of epoetin alfa, epoetin beta and darbepoetin alfa in anaemia associated with cancer, especially that attributable to cancer treatment. Health Technol. Assess. 11(13), 1-202 (2007).

32. Tang, W. et al. Diagnostic accuracy of tests to detect hepatitis $\mathrm{C}$ antibody: A meta-analysis and review of the literature. BMC Infect. Dis. 17(1), 695 (2017).

33. Freiman, J. M. et al. Hepatitis $C$ core antigen testing for diagnosis of hepatitis $C$ virus infection: A systematic review and metaanalysis. Ann. Intern. Med. 165(5), 345-355 (2016).

34. Organization WH. CHOosing Interventions that are Cost Effective (WHO-CHOICE). Available from: https://www.who.int/choice/ toolkit/en/. (last accessed 21 November 2018).

35. McAdam-Marx, C. et al. All-cause and incremental per patient per year cost associated with chronic hepatitis $\mathrm{C}$ virus and associated liver complications in the United States: A managed care perspective. J. Manag. Care Pharm. 17(7), 531-546 (2011).

36. The Global Fund. Highest-ever funding allocations announced to fight AIDS, TB and malaria. 2019. Available from: https://www. theglobalfund.org/en/news/2019-12-18-highest-ever-funding-allocations-announced-to-fight-aids-tb-and-malaria/. (last accessed 31 January 2020).

37. Radley, A. et al. A systematic review and meta-analysis of community and primary-care-based hepatitis $\mathrm{C}$ testing and treatment services that employ direct acting antiviral drug treatments. BMC Health Serv. Res. 19(1), 765 (2019).

\section{Acknowledgements}

We thank Yvan Hutin of WHO Global Hepatitis Program for his guidance, our partners in Georgia; Georgian Ministry of Health, National Centers for Disease Control, Georgian Harm Reduction Network and all study participants and staff from the study teams.

\section{Author contributions}

Study concept and design: M.A., Y.Z., H.Z., R.A., S.S., J.C. Statistical analysis: M.A., Y.Z., H.Z., T.Z., H.Z. Interpretation of data: all authors. Drafting of manuscript: M.A., Y.Z., S.S., J.C. Critical revision of the manuscript for important intellectual content: all authors.

\section{Funding}

This study was supported in parts by a grant from Unitaid as part of the HEADStart project, and the National Science Foundation award 1722665. The funding sources had no role in the design and conduct of the study; collection, management, analysis, and interpretation of the data; preparation, review, or approval of the manuscript; and decision to submit the manuscript for publication. 


\section{Competing interests}

S. Shilton is an employee of The Foundation for Innovative New Diagnostics. Dr. Chhatwal reported receiving grants from the National Science Foundation and Unitaid during the conduct of the study, grants and personal fees from Gilead and Merck \& Co outside the submitted work, and served as a partner with Value Analytics Labs outside the submitted work. All other authors have no competing/conflict of interest.

\section{Additional information}

Supplementary Information The online version contains supplementary material available at https://doi.org/ 10.1038/s41598-021-00362-y.

Correspondence and requests for materials should be addressed to J.C.

Reprints and permissions information is available at www.nature.com/reprints.

Publisher's note Springer Nature remains neutral with regard to jurisdictional claims in published maps and institutional affiliations.

(c) (i) Open Access This article is licensed under a Creative Commons Attribution 4.0 International License, which permits use, sharing, adaptation, distribution and reproduction in any medium or format, as long as you give appropriate credit to the original author(s) and the source, provide a link to the Creative Commons licence, and indicate if changes were made. The images or other third party material in this article are included in the article's Creative Commons licence, unless indicated otherwise in a credit line to the material. If material is not included in the article's Creative Commons licence and your intended use is not permitted by statutory regulation or exceeds the permitted use, you will need to obtain permission directly from the copyright holder. To view a copy of this licence, visit http://creativecommons.org/licenses/by/4.0/.

(C) The Author(s) 2021, corrected publication 2022 